\title{
P 157 AUDIT OF PRESCRIBING OF OPIATES AND SEDATIVES AT END OF LIFE IN A LARGE DISTRICT GENERAL HOSPITAL
}

Jacqueline Phillips, Beata LeBon, Nicola Jenkins. Frimley Park Hospital NHS Foundation Trust, Frimley, UK

\subsection{6/bmjspcare-2014-000654.198}

Background The review of the LCP included concerns that opiates and sedatives had been used inappropriately at end of life. Although studies of prescribing for hospice inpatients concluded safe practice and lack of evidence that their use shortens survival, there is a lack of studies examining similar aspects of prescribing in hospital inpatients.

Aims The aims of the audit were to:

Examine PRN and regular doses of opiates and sedatives in a sample of dying hospital inpatients

Examine the appropriateness of incremental increases of both drugs and their final doses

Compare the findings with the studies examining the above practice in hospice inpatients.

Methods Thirty deaths and their LCP documentation were audited retrospectively. The following data were collated: length of time on the LCP, specialist palliative care involvement and PRN and regular opiate and sedative doses in the last 7 days of life and their incremental increases.

Results The average use of the LCP was 2.9 days. Opioid analgesia was administered to $73.3 \%$ patients $(46.7 \%$ receiving it via a CSCI.) The mean daily morphine equivalent doses (MDMED) for days 1, 2, 3, 4, 5, 6, 7 before death were 22.3, $21.8,22.7,23.7,23.6,22.5$ and $20.8 \mathrm{mg}$ respectively (range $2.5-80 \mathrm{mg}$ over $24 \mathrm{hrs}$; one dose of $80 \mathrm{mg}$ skewing the results).

Sedation (with midazolam, haloperidol or both) was provided in $70 \%$ cases $(43 \%$ via a CSCI). In patients who received midazolam in a CSCI the mean daily doses on day $1,2,3,4,5,6,7$ before death were $9.1,8.3,7.3,11.3,8.8,5.0$ and $3.8 \mathrm{mg}$ respectively.

Conclusion The audit concluded safe use of opiates and sedatives with incremental increases justified by the PRN doses and documented symptoms with no evidence of doses being unduly accelerated during the LCP use. The final doses of both drugs were lower than those reported by some hospice based audits. 